\title{
Exotic searches at NA62
}

\author{
Roberta Volpe ${ }^{1}$ \\ for the NA62 Collaboration \\ ${ }^{1}$ Université Catholique de Louvain, Louvain-La-Neuve, Belgium
}

\begin{abstract}
The NA62 Experiment, thanks to the high intensity proton beam provided by the SPS, is a suitable apparatus to search for Beyond Standard Model (BSM) scenarios feebly coupled to SM, manifesting itself at the MeV$\mathrm{GeV}$ energy range. In this document a report of Heavy Neutral Lepton and Dark Photons searches results and the prospect for other BSM searches at NA62 are given.
\end{abstract}

\section{Introduction}

It is well known that the Standard Model (SM) is not complete, given that it is not able to explain several observations: Dark Matter, Baryon asymmetry of the Universe, neutrino masses, the $g-2$ anomaly. Besides looking for new particles at higher and higher energies, what is called energy frontier, a promising complementary strategy consists in searching for new physics sectors feebly coupled to the SM which could have eluded the LHC experiments because of their tiny couplings. These BSM scenarios could manifest themselves even at low-moderate energy. In order to detect the effects of very small couplings a large amount of interactions is needed, therefore a high intensity beam and fixed target experiments are particularly suitable. While new experiments dedicated to these exotic searches are being proposed, the already running NA62 experiment can be exploited to this purpose. The NA62 Experiment has been designed and constructed keeping in mind the requirements to perform a precise measurement of $K^{+} \rightarrow \pi^{+} \nu \bar{v}$ [1]. A $400 \mathrm{GeV}$ proton beam impinges a Berillium target giving rise to a secondary beam which, after passing through a system of collimators and magnets, results constituted by monochromatic $\sim 75 \mathrm{GeV}$ positively charged particles, of which only $6 \%$ are kaons. The NA62 apparatus is shown in Fig.1 and is described in detail in [2]. The Kaons are identified by a Cherenkov counter (Cedar or KTAG) and tracked by a kaon spectrometer (Gigatracker or GTK). The decay fiducial volume is $60 \mathrm{~m}$ long, the momentum and direction of the charged decay products are measured by a spectrometer (STRAW). The particle identification is provided by a Ring Imaging Cherenkov detector $(\mathrm{RICH})$ and a system of calorimeters: the hadronic calorimeters, aimed to veto muons, and a electromagnetic calorimeter ( $\mathrm{LKr}$ ) which detects and measures photon energies. Photons at any angles are vetoed by an hermetic system of detectors. NA62 is ending its first period of data taking (2015-2018) before the Long Shutdown 2 (LS2). With the collected data several analyses looking for exotic particles are ongoing and few of them have been published. In Sec. 2 and Sec. 3 the results of a search for Heavy Neutral Lepton and a search for Dark Photon, respectively, are reported. An overview of other ongoing analyses and prospects is given in Sec. 4. 


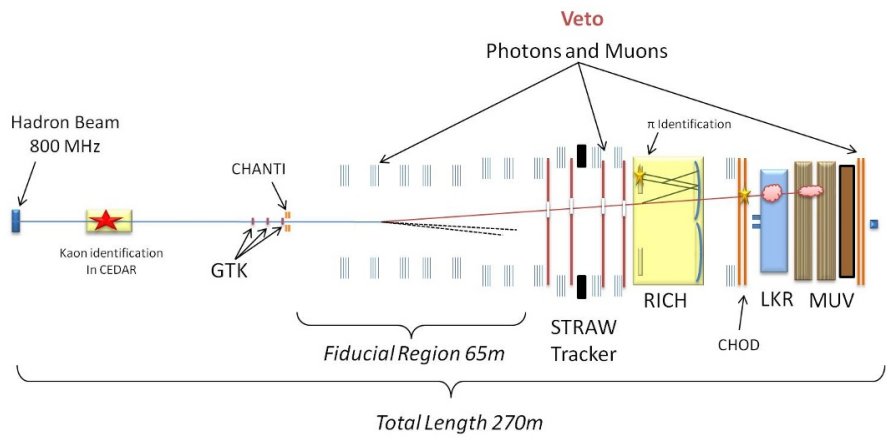

Figure 1. Layout of NA62 Experiment at CERN.

\section{Heavy Neutral Lepton search}

The Neutrino Minimal Standard Model ( $v$ MSM) [3] is a natural SM extension introducing the existence of three right-handed singlet heavy neutrinos (HNL) which mix with the standard ones and explain simultaneously neutrino oscillations, dark matter and baryon asymmetry in the Universe. According to $v$ MSM HNL, in the following indicated with $N$, with mass below the kaon mass can be produced through mixing with SM neutrinos in the leptonic kaon decays $K^{+} \rightarrow l^{+} N$, where $l=e, \mu$. The branching ratio of those decays can be expressed as:

$$
B R\left(K^{+} \rightarrow l^{+} N\right)=B R\left(K \rightarrow l^{+} v\right) \cdot \rho_{l}\left(m_{M}\right) \cdot\left|U_{l 4}\right|^{2},
$$

where: $B R\left(K \rightarrow l^{+} v\right)$ is the branching ratio of the SM decay, $\rho_{l}\left(m_{M}\right)$ is a kinematic factor accounting for helicity suppression and phase space dependence on $m_{N}$ [4][5] and $\left|U_{l 4}\right|$ is the mixing parameter between $N$ and $v$. Assuming $\left|U_{l 4}\right|^{2}$, the mean free path of HNL before decaying to SM particles is above $10 \mathrm{Km}$ and the probability that it interact with the NA62 detectors is negligible. Therefore the analysis selection requires the presence of a lepton, $e$ or $\mu$ and nothing more. The analysis then proceeds through a search for a peak in the squared missing mass $m_{\text {miss }}^{2}=\left(P_{K}-P_{l}\right)^{2}$ variable. The SM decays $K \rightarrow l^{+} v, N_{K}^{l}$, are used as normalization, therefore, calling $A_{l}^{N}$ the acceptance for the BSM decays, the number of selected events $N_{S}$ can be written as:

$$
N_{S}=N_{K}^{l} \cdot B R\left(K \rightarrow l^{+} v\right) \cdot A_{l}^{N},
$$

The base of the event selection is common for SM and BSM events and shortly described in the following. A sample of 2015 data taking, collected with a minimum-bias trigger and corresponding to $3 \cdot 10^{8}$ kaon decays, has been used. During that period the GTK was still in commissioning phase, therefore the nominal kaon momentum has been used. Single positively-charged tracks within the detector acceptance, with momenta between 5 and 70 $\mathrm{GeV}$, are selected in the spectrometer. The events must have a well reconstructed kaon decay vertex to one single particle and no photons are allowed. The selected downstream track, if with momentum $p<40 \mathrm{GeV}$, is required to be associated to a RICH candidate and identified as a $\mu^{+}$or $e^{+}$. Further particle identification is provided by the ratio $E / p$, where $E$ is the energy deposited in the $\mathrm{LKr}$, and the information provided by the hadronic calorimeters. The distributions of $m_{m i s s}^{2}$ for the two samples selected as $K^{+} \rightarrow e^{+} v$ and $K^{+} \rightarrow \mu^{+} v$ are shown in Fig.2. The squared missing $m_{m i s s}^{2}$ is computed with the lepton 3-momentum of the spectrometer track in the corresponding mass hypothesis and with the beam average 3momenta monitored from reconstructed $K^{+} \rightarrow \pi^{+} \pi^{+} \pi^{-}$events. Signals from the SM leptonic 
decays are observed as peaks at $m_{\text {miss }}^{2} 0$ with resolutions of $4.9(4.7) \times 10^{3} \mathrm{GeV}^{2}$ in the $e^{+}\left(\mu^{+}\right)$ case. Two different signal regions have been defined for the two final states. For the $e$-channel
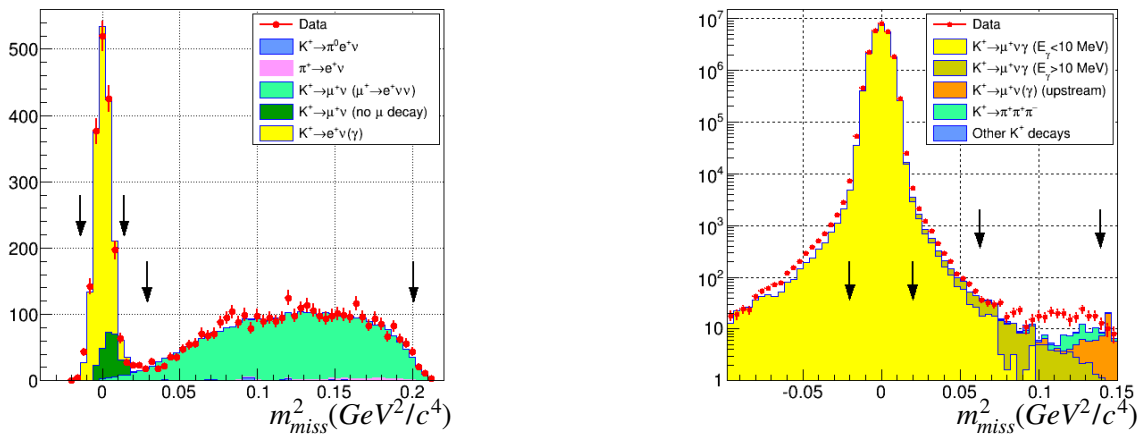

Figure 2. Distributions of $m_{\text {miss }}^{2}$ for data and simulated events passing the (left) $e^{+}$and (right) $\mu^{+}$ selections (bin widths of $0.004 \mathrm{GeV}^{2}$ ). Pairs of vertical lines in each plot indicate the boundaries of the $\mathrm{SM}$ and HNL signal regions.

the number of SM events in the signal region $170-448 \mathrm{MeV}$ is $N_{K}^{e}=(3.00 \pm 0.11) \times 10^{8}$ kaon leptonic decays into electrons. The $\mu$-channel signal region is $250-373 \mathrm{MeV}$ and the number of observed SM events is $N_{K}^{\mu}=1.06 \pm 0.02 \times 10^{8}$. The search for HNL proceeds with a bump hunting over a smooth background estimated directly from data. The procedure is valid provided that there are no peaking background structures in the $m_{m i s s}^{2}$ signal region and this is guaranteed by MC checks. A scan on the $m_{m i s s}^{2}$ has been performed, with $1 \mathrm{MeV}$ step size. Per each $m_{N}$ hypothesis a window of $1.5 \times \sigma\left(m_{N}\right)$ has been considered, where $\sigma\left(m_{N}\right)$ is the HNL mass resolution evaluated with MC simulations and depends on the value of $m_{N}$ and runs in the range $\sim 1-18 \mathrm{MeV}$. Per each window a counting experiment upper limit has been obtained, with the background and its uncertainty evaluated from sidebands and the expected signal from Eq. (1) and Eq. (2). Fig. 3 compares the upper limits on $\left|U_{l}^{4}\right|^{2}$ at $90 \%$ C.L. set by NA62 as a function of HNL mass with the results from other HNL production searches.

\section{Dark photon search}

In a general set of hidden sector models with an extra $U(1)$ gauge symmetry, the interaction of a dark photon (DP or $A^{\prime}$ ) with the visible sector proceeds through kinetic mixing with the SM hypercharge [6][7]. In some models the DP can decay soon after production in the final state, in others it is long-lived, namely it moves in the experimental apparatus before decaying to a visible final state or it decays to invisible particles. The two latter scenarios have been investigated at NA62 considering the decay: $K^{+} \rightarrow \pi^{+} \pi^{0}$, with $\pi^{0} \rightarrow \gamma A^{\prime}$ and $A^{\prime} \rightarrow$ inv, meaning the search proceeds through bump hunting in the square missing mass distribution $m_{\text {miss }}^{2}=\left(P_{K}-P_{\pi}-P_{\gamma}\right)^{2}$. The analysis has been performed with a sample of $1.5 \times 10^{10}$ $K^{+}$decays, corresponding to about $5 \%$ of the dataset collected in 2016. Events are selected requiring a single downstream track reconstructed in the spectrometer and matched with the GTK in a vertex in the fiducial volume. The track is identified as a positive pion by the RICH and calorimeter system, a single photon cluster in the LKr calorimeter is reconstructed, and no other activity is detected. The $m_{\text {miss }}^{2}$ distribution is expected to peak around the $A^{\prime}$ mass for the $\pi^{0} \rightarrow \gamma A^{\prime}$ decay and around zero for the background process $\pi^{0} \rightarrow \gamma \gamma$ with one photon undetected. For each $m_{A}$, the signal region is defined as a 1.5 standard deviation range around the expected invariant mass peak and the expected background and its uncertainty are 
evaluated from the data in the sidebands. A counting experiment upper limit is derived for the kinetic mixing mixing parameter $\epsilon$ and it is shown in Fig.3.

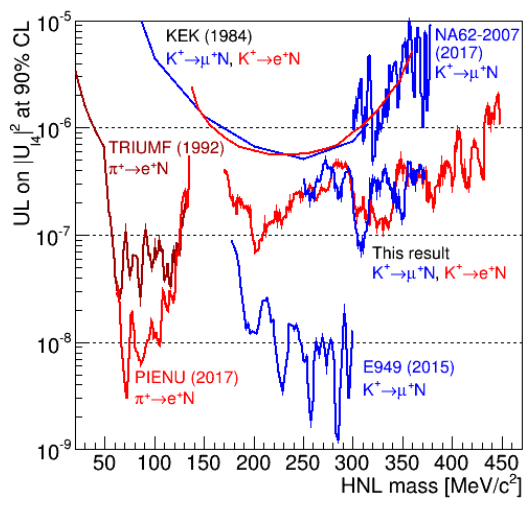

Figure 3. $90 \%$ C.L. Upper Limit (UL) in the plane of coupling $\left|U_{4 l}\right|$ versus mass.



Figure 4. The $90 \%$ CL exclusion limit on the kinetic mixing parameter versus the mass of the Dark Photon.

\section{Prospects for further analyses}

Large improvements of the NA62 searches for HNL are expected soon from the analysis of the full data set (2016-2018). Not only a larger data set will be available due to higher beam intensity and longer running periods, but the analysis performance will benefit also from the use of GTK which will improve HNL mass resolution and background reduction. Several other searches for exotic particles are ongoing. In order to search for new physics particles with mass larger than the Kaon mass, $B$ and $D$ meson decays are also considered. The production of $B$ and $D$ can arise from the interaction of the protons of the primary SPS beam with the nucleons of the $\mathrm{Cu}$ collimators (indicated with TAX in Fig.1). Large data samples can be obtained with two different approaches: $(i)$ using a trigger which does not require the presence of kaons, (ii) by removing the Be target and closing the collimators aperture. The approach $(i)$ is limited by the background contribution from the Kaon decays. Instead (ii) is limited by the statistics: it requires a change in the beamline which prevents the collection of data for the $\pi v \bar{v}$ analysis, the main goal of the experiment, therefore extended periods of this kind of data taking will be possible only after the $\pi v \bar{v}$ program is complete. Searches for HNL and Dark Photons with visible decays, Dark Scalars, Axion Like Particles will be performed in the next years. The large samples of kaon and pion decays in NA62 provide an opportunity also to perform searches for a range of novel phenomena and forbidden $K^{+}$decay modes with an unprecedented precision, improving the current limits in the searches for Lepton Number Violation (LNV), Lepton Flavor Universality Violation (LFUV) and Lepton Flavor Violation (LFV) decays. These searches are not limited by backgrounds and sensitivity down to $10^{-11}$ can be reached.

\section{References}

[1] N. Lurkin et al. [NA62 Collaboration], these proceedings.

[2] E. Cortina Gil et al. [NA62 Collaboration], JINST 12, no. 05, P05025 (2017)

[3] A. Boyarsky et al., Ann. Rev. Nucl. Part. Sci. 59, 191, (2009) 
[4] R. E. Shrock, Phys. Lett. B 96, 159 (1980)

[5] R. E. Shrock, Phys. Rev. D 24, 1232 (1981)

[6] L. B. Okun, Sov. Phys. JETP 56, 502 (1982)

[7] B. Holdom, Phys. Lett. B 166, 196 (1986)

[8] D. Banerjee et al. [NA64 Collaboration], Phys. Rev. Lett. 118, no. 1, 011802 (2017) 